\title{
How to Avoid Facial Nerve Injury in Mastoidectomy?
}

\author{
Nam-Gyu Ryu and Jin Kim \\ Department of Otorhinolaryngology, Inje University College of Medicine, Ilsan Paik Hospital, Goyang, Korea
}

Received November 30, 2015

Revised January 29, 2016

Accepted February 13, 2016

\author{
Address for correspondence \\ Jin Kim, $\mathrm{MD}, \mathrm{PhD}$ \\ Department of Otorhinolaryngology, \\ Inje University College of Medicine, \\ Ilsan Paik Hospital, \\ 170 Juhwa-ro, Ilsanseo-gu, \\ Goyang 10380, Korea \\ Tel +82-31-910-7114 \\ Fax +82-31-910-7518 \\ E-mail jinsound@gmail.com
}

\begin{abstract}
Unexpected iatrogenic facial nerve paralysis not only affects facial disfiguration, but also imposes a devastating effect on the social, psychological, and economic aspects of an affected person's life at once. The aims of this study were to postulate where surgeons had mistakenly drilled or where obscured by granulations or by fibrous bands and to look for surgical approach with focused on the safety of facial nerve in mastoid surgery. We had found 14 cases of iatrogenic facial nerve injury (IFNI) during mastoid surgery for 5 years in Korea. The medical records of all the patients were obtained and analyzed injured site of facial nerve segment with surgical technique of mastoidectomy. Eleven patients underwent facial nerve exploration and three patients had conservative management. $43 \%$ (6 cases) of iatrogenic facial nerve injuries had occurred in tympanic segment, $28.5 \%$ (4 cases) of injuries in second genu combined with tympanic segment, and $28.5 \%$ (4 cases) of injuries in mastoid segment. Surgeons should try to identify the facial nerve using available landmarks and be kept in mind the anomalies of the facial nerve. With use of intraoperative facial nerve monitoring, the avoidance of in order to avoid IFNI would be possible in more cases. Many authors emphasized the importance of intraoperative facial nerve monitoring, even in primary otologic surgery. However, anatomical understanding of intratemporal landmarks with meticulous dissection could not be emphasized as possible to prevent IFNI.
\end{abstract}

J Audiol Otol 2016;20(2):68-72

\section{Introduction}

Iatrogenic injury to the facial nerve is one of the most devastating complications of ear surgery. Some authors have found the incidence of facial nerve injury in primary otologic procedures to be as high as $1 \%$ and $4 \%$. But in revision procedure, it was reported to be more than double when compared to primary operations at $4 \%$ to $10 \%$ and $0.6 \%$ to $3.7 \%$, respectively $[1,2]$. Sudden and unexpected facial nerve paralysis not only affects facial appearance or diminished facial expression, but also imposes a devastating effect on the social, psychological, and economic aspects of an affected person's life at once [3,4]. Recently, we had found 14 cases of iatrogenic facial nerve injury during mastoid surgery for 5 years in Korea. The medical records of all the patients were

This is an Open Access article distributed under the terms of the Creative Commons Attribution Non-Commercial License (http://creativecommons.org/licenses/by-nc/3.0/) which permits unrestricted non-commercial use, distribution, and reproduction in any medium, provided the original work is properly cited. obtained and analyzed injured site of facial nerve segment with surgical technique of mastoidectomy (Table 1).

Eleven patients underwent facial nerve exploration and three patients had conservative management. Cases of pre-operative facial nerve paralysis due to chronic otitis media and/or cholesteatoma were excluded as these cases are managed differently from cases of unexpected iatrogenic injury. $43 \%$ (6 cases) of iatrogenic facial nerve injuries had occurred in tympanic segment, $28.5 \%$ (4 cases) of injuries in second genu combined with tympanic segment, and $28.5 \%$ (4 cases) of injuries in mastoid segment (Fig. 1).

Despite technological advances, such as the introduction of the operating microscope and delicate surgical drill, and the availability of pre-operating imaging, the overall risk of iatrogenic facial nerve paralysis remains considerably high. Hohman, et al. [5] reported otologic surgery resulted in $17 \%$ of all postoperative facial nerve palsy. Especially, $82 \%$ of otologic facial nerve injuries were caused by mastoid surgery.

Identifying the facial nerve is fundamental to performing 
Table 1. Summary 14 patients with postoperative facial nerve injury

\begin{tabular}{|c|c|c|c|c|c|}
\hline No. & Disease & Type of surgery & Surgical procedure & Site of injury (segments) & $\begin{array}{c}\text { Postoperative facial } \\
\text { function }\end{array}$ \\
\hline 1 & SNHL & $\mathrm{Cl}(\mathrm{CWU})$ & Posterior tympanotomy & Mastoid & H-B Gr. V \\
\hline 2 & SNHL & $\mathrm{Cl}(\mathrm{CWU})$ & Posterior tympanotomy & Mastoid & H-B Gr. V \\
\hline 3 & SNHL & $\mathrm{Cl}(\mathrm{CWU})$ & Posterior tympanotomy & Mastoid & H-B Gr. III \\
\hline 4 & Cholesteatoma & CWD & Cholesteatoma removal & Tympanic & H-B Gr. V \\
\hline 5 & Cholesteatoma & CWD (revision) & Cholesteatoma removal & Tympanic & H-B Gr. V \\
\hline 6 & Cholesteatoma & CWD (revision) & Drilling of facial nerve & $2^{\text {nd }}$ genu, tympanic & H-B Gr. VI \\
\hline 7 & Cholesteatoma & CWU & Pull injury of facial nerve & Tympanic & H-B Gr. V \\
\hline 8 & Cholesteatoma & CWU & Drilling of facial nerve & $2^{\text {nd }}$ genu, tympanic & H-B Gr. V \\
\hline 9 & $\mathrm{COM}$ & CWU & Drilling of facial nerve & Tympanic & H-B Gr. V \\
\hline 10 & $\mathrm{COM}$ & CWU & Drilling of facial nerve & Tympanic & H-B Gr. V \\
\hline 11 & COM & CWU & Drilling of facial nerve & $2^{\text {nd }}$ genu, tympanic & H-B Gr. V \\
\hline 12 & COM & CWU & Failure of finding antrum & $2^{\text {nd }}$ genu, tympanic & H-B Gr. VI \\
\hline 13 & $\mathrm{COM}$ & CWU & Drilling of facial nerve & Mastoid & H-B Gr. III \\
\hline 14 & COM, microtia & CWU & Drilling of facial nerve & Tympanic & H-B Gr. III \\
\hline
\end{tabular}

SNHL: sensorineural hearing loss, Cl: cochlear implantation, $\mathrm{H}-\mathrm{B}$ : House-Brackmann grade, CWU: canal wall up mastoidectomy, CWD: canal wall down mastoidectomy, COM: chronic otitis media

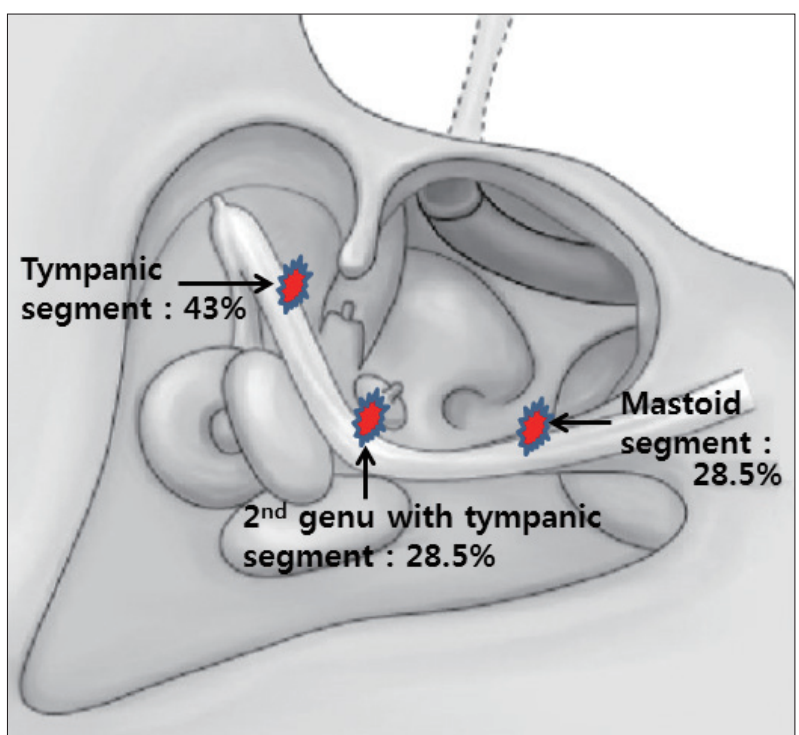

Fig. 1. Injured segments of facial nerve during otologic surgery in Korea.

good mastoid surgery. In intact canal wall mastoidectomy (or when a cochlear implant is placed), the space between the facial nerve and the chorda tympani nerve (the facial recess) provides access into the middle ear. In canal wall down approaches, identifying the facial nerve allows the surgeon to lower the facial ridge appropriately, creating a more care-free cavity and/or preventing the recurrence in case of cholesteatoma.

The aims of this study were to postulate where surgeons had mistakenly drilled or where obscured by granulations or by fibrous bands and to look for surgical approach with focused on the safety of facial nerve in mastoid surgery.

\section{Facial Nerve Secured Mastoidectomy}

The landmarks for skeletonize of intra-temporal facial nerve are divided into major landmarks and minor landmarks. The major landmarks include the lateral semicircular canal, the short process of the incus, and the posterior bony external auditory canal. The minor landmarks include the digastric ridge, the cochleariform process, and the oval window. The major landmarks should always be exposed for mastoidectomy and the minor landmarks could be exposed if necessary.

Surgeon can confuse the location of facial nerve in cases of revision mastoidectomy, brittle mastoid such as fibrous dysplasia, contracted mastoid, huge cholesteatoma, and anatomical variation of facial nerve. In revision surgery which had been destructed by previous surgery, there can be absence of any landmarks for identification of facial nerve.

\section{Exact localization of the antrum by starting with wide exposure}

A key landmark in performing mastoid surgery is the antrum with the dome of the horizontal semicircular canal along its floor. The ease of locating the antrum depends largely on the degree of mastoid pneumatization. An exact localization of the antrum is a fundamental procedure for disease control on middle ear without any damage of facial nerve. Since the antrum is very consistent and there is no important structure lateral to it, the antrum serves as one of the most important landmarks in the initial stage of mastoidectomy [6]. Two grooves are drilled along the superior and posterior bony external auditory canal canal wall to find antrum. The antrum 
is located at the intersection of both grooves [7]. The initial burr cut is made along the linea temporalis, which marks lowest points of the middle fossa dura in most cases. The second burr cut is along a line perpendicular to the one just described and tangent to the posterior margin of the ear canal. The apex of this triangle is directly over the lateral semicircular canal (LSCC) [8]. Failure to accurate location of antrum and subsequent further drilling inferiorly and medially may also risk drilling into LSCC and causing labyrinthine fistula.

\section{A constant thickness of posterior bony external canal}

Adequate bony exposure of superior part of external canal is helpful to expose the attic region, which serves as a good guide to the direction and depth of the antrum and attic. Once the attic and its contents have been identified, further drilling should be done posteroinferiorly to find the aditus, short process of incus, LSCC and second genu of the facial nerve [9].

\section{Surgeon tends to drill mistakenly more inferiorly and posteriorly to the actual site of antrum}

Drilling more inferiorly and posteriorly to the actual site of antrum could cause the injury of facial nerve at the second genu. The antrum could also have been obscured by granulations or by fibrous bands in second genu. Therefore, the identification of the antrum, the surgeon needs to start drilling strictly over the MacEwan's triangle and then enlarge the mastoid bowel [9]. If the surgeon still fails to identify the antrum, drilling more anterosuperiorlytoward attic can be suggested [9].

\section{Can we trust the sitting of mastoid segment in intra-temporal drilling?}

In cadaver study, the mean length of mastoid segment of facial nerve was about $15 \mathrm{~mm}$ which was lying lateral to the stapedius muscle and the sinus tympani. Both of the mean distance at the level of the posterior border of the oval window and facial nerve, and from and the round window to the facial nerve were $4 \mathrm{~mm}$. The lower one third of the mastoid segment was anteromedial to the digstic ridge, which was ranged from 2 to $5 \mathrm{~mm}$. The course of the mastoid segment showed variations in its descent from the second genu to stylomastoid formaen. The course of the vertical or mastoid segment of the facial nerve showed variations in its descent from the second genu to the stylomastoid foramen. In $60 \%$ of specimens, it was showing a vertical descent upto the stylomastoid foramen, in $20 \%$ each the nerve was observed to descend slightly laterally or medially [10].

The mastoid segment of the nerve courses near the line between the short process of the incus and the digastrics ridge, and in the posterior wall of the tympanic cavity. The bone is removed from the stylomastoid foramen to the tympanic segment of facial nerve until the nerve becomes visible under the last egg shell of bone [7]. Surgeons should always be prepared to identify the nerve at any segment using available landmarks and be kept in mind the anomalies of the facial nerve. The facial nerve may be located inferiorly to the oval window, or may be divided into branches [6]. Moreover, relations to various structures like cochleariform process, pyramidal eminence, LSCC, round window, oval widow and digastrics ridge can be also variable [10].

\section{Finding of intratemporal facial nerve}

The total length of the facial nerve in the temporal bone is around $30 \mathrm{~mm}$. Within the temporal bone, the nerve is divided into three segments. The labyrinthine segment runs laterally from the fundus of the internal auditory canal until it reaches the geniculate ganglion. The geniculate ganglion is located immediately superior to the cochlea, beneath the middle fossa plate [6]. The tympanic portion of facial nerve is identified along the inferior margin of the LSCC [7]. The prominence of the LSCC is one of the most important landmarks for the facial nerve, running from anterosuperior to posteriorinferior. Tympanic segment may be dehiscent, and the facial nerve may be exposed to the tympanic cavity. Around the posterior edge of the oval window and medial to the short process of the incus, the facial nerve follows a gentle curve in an inferior direction, which is called the second genu [6]. The rotation of the burr is reversed when working along the tympanic and labyrinthine segments of the facial nerve [7].

The course of the mastoid segment of the facial canal is identified through the bone using the monitoring of facial nerve (NIM-II) [7]. The mastoid segment runs nearly vertically toward the stylomastoid foramen [6]. As the facial nerve travels inferiorly in its course to the stylomastoid foramen, it travels in a slightly posterior direction and also travels laterally [8]. Skeletonization of mastoid segment of the facial canal is done in a retrograde fashion beginning from the stylomastoid foramen. The digastric ridge and stylomastoid periosteum are exposed to identify the stylomastoid foramen [7]. A further landmark for the mastoid segment is the inferior edge of the posterior semicircular canal (PSCC). The pyramidal segment of the facial nerve is situated $2 \mathrm{~mm}$ anteriorly and laterally to the inferior edge of the PSCC $[7,8]$.

There are two things one may notice when approaching the facial nerve. Frequently, bleeding is encountered from vessels intimately associated with, but lying outside the bony canal of the facial nerve. Before actually uncovering the nerve, one may note its white sheath showing through the thin bone [8]. 


\section{Variant courses of facial nerve}

Among the factors that influence injury to the facial nerve, the condition of facial canal is very important [11]. Dehiscence of the facial canal would increase the risk of facial nerve injury, even in the routine ear surgery [12]. The main cause of facial canal dehiscence is variation of the normal developmental process, which is followed by longstanding inflammation, prior ear surgery or trauma, and the pressure effect of tumorous lesions such as cholesteatoma [13-15]. The incidence of intraoperative facial canal dehiscence was reported from $0.5 \%$ to $74 \%$ by literatures [11]. The most common site of iatrogenic facial nerve palsy during mastoid surgery was at the second genu [9]. Previous study reported the incidence of facial nerve dehiscence during the primary operation to be $88 \%$ in the tympanic segment while $12 \%$ at the mastoid segment among the cases of facial nerve dehiscence [16].

In the tympanic segments, vulnerability of the facial nerve is associated with a high percent of dehiscence of facial canal $[16,17]$. The facial nerve may lie over to varying degrees, bifurcate around or even run below the level of the oval window [9].

\section{Discussion}

In conducting of drilling the mastoid for the elimination of any otologic disease, the surgeon can unexpectedly encounter any kinds of difficulties. It results from a distorted anatomy of the mastoid by many otologic lesions including destructing cholesteatoma, bony atrophied contraction, plunging granulation, or revised surgery. Another can result from anomalies and variations in the temporal bone anatomy (dehiscence of the facial nerve, poorly aerated mastoid, postmatoiditis mastoid) [18].

In order to avoid iatrogenic facial nerve injury (IFNI), surgeon should be basically familiar with the normal course of the facial nerve, also with any anatomical variations that may be encountered in congenital anomalies, prior surgery, or trauma [5]. Exact ascertain of the facial nerve throughout mastoid dissection in any planes could prevent IFNI undoubtedly.

Intraoperative facial nerve monitoring during otologic surgery was not used routinely in 1990s. Roland and Meyerhoff [19] reported that only 4\% of respondents from members of American Otological and Neurolotology Society agreed that facial nerve monitoring should be used in all tympanomastoid surgery. In contrast, facial nerve monitoring is now routinely used in most cases involving retrocochlear lesion, and at least some parotid and chronic ear surgeries. $\mathrm{Hu}$, et al. [20] reported about half of respondents of their study believed that facial nerve monitoring should be considered in chronic ear surgery, especially in younger surgeons.

However, the use of the intraoperative facial nerve monitoring will certainly help the surgeon identify the facial nerve by an early warning signal of potential injury, it can not be substituted for thorough anatomical knowledge of the temporal bone $[9,20]$. Conscientious surgical technique with direct visualization of facial nerve branches should be emphasized for avoiding IFNI [5]. In other words, intraopertavie facial nerve monitor can add another level of safety with a complication that we otherwise make every effort to avoid [21].

Although high resolution computed tomography (CT) of temporal bone can show the extent of the disease and guide the procedures performed during the previous surgery for revision mastoid surgery [9], preoperative CT in primary mastoid surgery would not have much benefit in avoiding facial nerve injury.

In patients with IFNI, patients should be managed as soon as possible, in whom explorative surgery is performed, to regain significant recovery of facial function [5]. Fundamentally, surgeons operating around the facial nerve who encounter unexpected postoperative facial palsy may refer patients to a facial nerve clinic to be any useful options, particularly if unfamiliar with microsurgical repair techniques [5].

\section{Conclusion}

IFNI is considered as one of the most devastating events of otologic surgery and one of frequent causes for medical litigation. IFNI affects not only facial expression but psychosocial effects, both the patients and surgeons. There have been reported major and minor anatomical landmarks for facial nerve in literatures. Surgeons should try to identify the facial nerve using available landmarks and be kept in mind the anomalies of the facial nerve. With use of intraoperative facial nerve monitoring, the avoidance of IFNI would be possible in more cases. Many authors emphasized the importance of intraoperative facial nerve monitoring, even in primary mastoidectomy operations. However, anatomical understanding of intratemporal landmarks with meticulous dissection could not be emphasized as possible to prevent IFNI.

\section{Conflicts of interest}

The authors have no financial conflicts of interest.

\section{REFERENCES}

1) Harner SG, Daube JR, Ebersold MJ. Electrophysiologic monitoring of facial nerve during temporal bone surgery. Laryngoscope 1986; 96:65-9.

2) Wilson L, Lin E, Lalwani A. Cost-effectiveness of intraoperative fa- 
cial nerve monitoring in middle ear or mastoid surgery. Laryngoscope 2003;113:1736-45.

3) Bradbury ET, Simons W, Sanders R. Psychological and social factors in reconstructive surgery for hemi-facial palsy. J Plast Reconstr Aesthet Surg 2006;59:272-8.

4) VanSwearingen JM, Cohn JF, Turnbull J, Mrzai T, Johnson P. Psychological distress: linking impairment with disability in facial neuromotor disorders. Otolaryngol Head Neck Surg 1998;118:790-6.

5) Hohman MH, Bhama PK, Hadlock TA. Epidemiology of iatrogenic facial nerve injury: a decade of experience. Laryngoscope 2014;124: 260-5.

6) Sanna M, Sunose H, Mancini F, Russo A, Taibah A, Falcinoi M. Middle Ear and Mastoid Microsurgery. 2nd ed. New York: Thieme Publishing;2012.

7) Fisch U, May JS, Linder T. Tympanoplasty, Mastoidectomy, and Stapes Surgery. 2nd ed. New York: Thieme;2008.

8) Brackmann DE, Shelton C, Arriga MA. Otologic surgery. 2nd ed. Philadelphia: WB Saunders;2001.

9) Asma A, Marina MB, Mazita A, Fadzilah I, Mazlina S, Saim L. Iatrogenic facial nerve palsy: lessons to learn. Singapore Med J 2009; 50:1154-7.

10) Yadav SP, Ranga A, Sirohiwal BL, Chanda R. Surgical anatomy of tympano-mastoid segment of facial nerve. Indian J Otolaryngol Head Neck Surg 2006;58:27-30.

11) Kim CW, Rho YS, Ahn HY, Oh SJ. Facial canal dehiscence in the initial operation for chronic otitis media without cholesteatoma. Auris Nasus Larynx 2008;35:353-6.
12) Weber PC. Iatrogenic complications from chronic ear surgery. Otolaryngol Clin North Am 2005;38:711-22.

13) Bayazit YA, Ozer E, Kanlikama M. Gross dehiscence of the bone covering the facial nerve in the light of otological surgery. J Laryngol Otol 2002;116:800-3.

14) Djerić D, Savić D. Otogenic facial paralysis. A histopathological study. Eur Arch Otorhinolaryngol 1990;247:143-6.

15) Spector JG, Ge X. Ossification patterns of the tympanic facial canal in the human fetus and neonate. Laryngoscope 1993;103:1052-65.

16) Selesnick SH, Lynn-Macrae AG. The incidence of facial nerve dehiscence at surgery for cholesteatoma. Otol Neurotol 2001;22:12932.

17) Green JD Jr, Shelton C, Brackmann DE. Iatrogenic facial nerve injury during otologic surgery. Laryngoscope 1994;104(8 Pt 1):922-6.

18) Gragnaniello C, Kamel M, Al-Mefty O. Utilization of fluorescein for identification and preservation of the facial nerve and semicircular canals for safe mastoidectomy: a proof of concept laboratory cadaveric study. Neurosurgery 2010;66:204-7.

19) Roland PS, Meyerhoff WL. Intraoperative electrophysiological monitoring of the facial nerve: is it standard of practice? Am J Otolaryngol 1994;15:267-70.

20) Hu J, Fleck TR, Xu J, Hsu JV, Xu HX. Contemporary changes with the use of facial nerve monitoring in chronic ear surgery. Otolaryngol Head Neck Surg 2014;151:473-7.

21) Ruhl DS, Hong SS, Littlefield PD. Lessons learned in otologic surgery: 30 years of malpractice cases in the United States. Otol Neurotol 2013;34:1173-9. 